\title{
How to interfere with memory for sentence meaning
}

\author{
JUDITH ORASANU \\ Rockefeller University, New York, New York 10021 \\ and \\ ROSAMOND GIANUTSOS \\ Adelphi University, Garden City, Long Island, New York 11530
}

\begin{abstract}
Whether memory for the meaning of sentences is subject to disruption by a subsequent task and the extent of the disruption were investigated in the present study. Presentation and recall of 10-word test sentences were separated by the reading of 10 additional words that formed a 10 -word sentence, five 2-word sentences, or a random string. The additional words were presented simultaneously or as pairs. One group of subjects was also asked to recall the additional words after recalling the test sentence. Memory for test sentence meaning was not affected by syntactic structure or presentation format unless recall of the additional words was required. In this case, random strings interfered the most and 10-word sentences the least. The format variable interacted with structure. These results suggest that when recall is required, semantic processing continues beyond termination of the stimulus presentation. It is subject to disruption that varies with the processing requirements of the subsequent task.
\end{abstract}

Studies of short-term memory for sentences have yielded a highly reliable finding: Verbatim information from sentences is lost very quickly, but sentence meaning is accurately retained over a long period of time. Jarvella (1971) has shown that only the most recently presented clause is maintained verbatim and that verbatim information begins to be lost after as few as two additional words from a new clause (Jarvella, Snodgrass, \& Adler, 1978). Verbatim information is essentially unavailable after 20 additional syllables of related text, according to Sachs (1974). However, Jarvella reports good recall of the gist of clauses prior to the last one presented, and many studies using recognition procedures have found excellent retention of sentence meaning (Bransford \& Johnson, 1973; Kintsch, 1974; Sachs, 1974). While it is certainly the case that exact wording may be accurately retained over a long time (e.g., Kintsch \& Bates, 1977), this is not the usual case.

Studies that show such good retention of meaning have generally involved recognition procedures: People can recognize as new sentences that falsify originally presented information, but they cannot distinguish between paraphrases or inferences and original sentences. These studies show a high constant retention curve, suggesting that semantic analysis is complete and that the memory representation is very durable (Katz, 1973; Sachs, 1974). However, ability to detect semantically

Correspondence regarding this article should be sent to Judith Orasanu, National Institute of Education, 1200 19th Street N.W., Washington, D.C. 20208. false or inconsistent sentences does not necessarily imply that complete information is available for production in recall. Also, the high levels observed in recognition studies may reflect the construction of the test items.

The present study was designed to determine whether semantic analysis is subject to disruption by subsequent input, as reflected in recall, and whether the loss is a function of the processing demands of the subsequent input. At issue is whether semantic analysis is completed prior to displacement of the verbatim information on which it is based, or whether it continues beyond the clause or sentence boundary. If it continues, it may be subject to disruption.

The method selected in the present study paralleled that used to study verbatim memory: Presentation and recall of test sentences were separated by an interpolated task in which the amount of additional information to be processed was varied. Because verbatim recall was not desired, the interpolated material always contained 10 additional words, an amount expected to "displace" test sentences from short-term storage, given that the short-term memory span for sentences has been estimated at 10 words (Brener, 1940). Thus, recall would represent reconstruction from an abstract semantic representation rather than direct readout from an echoic short-term store.

The processing demands of the subsequent input were manipulated by varying the number of major syntactic units contained in the 10 words. They formed a single 10-word sentence, five 2-word sentences, or a random 
string. Most interference was expected from the random string, and least from the single 10-word sentence. This prediction derives from research on free recall of word lists, which showed recall from short-term storage to be a function of the number of subsequent items that are read in a fixed time interval following list presentation (Glanzer, Gianutsos, \& Dubin, 1969).

The prediction rests on the assumption that subjects will automatically unitize the 10 words based on their syntactic structure when they read them aloud, an assumption questioned by Aaronson and Shapiro(1976). They argued that syntactic unitization occurs only in memory tasks. For this reason, two studies were conducted, one that required recall of the subsequent material, and one that did not. In addition, two presentation formats were used in order to test the robustness of the unitizing process.

\section{METHOD}

\section{No Recall of Interpolated Material}

Subjects. Fourteen college students enrolled in introductory psychology classes participated in the study. They received class credit for their participation.

Materials. Forty eight 10 -word sentences varying in syntax and 60 2-word sentences were constructed. In addition, 12 10 -word random strings were generated by randomly selecting words from sentences used in a previous experiment. For each subject, 36 of the 4810 -word sentences were selected as test sentences; the remaining 12 sentences served as interpolated sentences. The pairing of interpolated materials with test sentences was randomized for each subject, as was the order of presentation of the items and conditions. Examples of a test sentence and the three types of interpolated materials are shown in Table 1.

The interpolated material was presented in one of two formats, either 10 words at a time (called simultaneous) or 2 words at a time (called successive). The 10 words of the test sentences were always presented simultaneously.

Design. Three types of interpolated materials (one 10-word sentence, five 2-word sentences, 10 random words) and two presentation formats (successive or simultaneous) were combined factorially, yielding six conditions that were varied within subjects. There were six trials in each of the six conditions, for a total of 36 trials, presented in block random order.

Procedure. All materials were presented on a memory drum designed to accept a computer printout. Test sentences were presented for $5 \mathrm{sec}$ each, as was the interpolated material in the simultaneous format. In the successive format, each 2 -word pair was presented for $1 \mathrm{sec}$, keeping the total presentation time constant at $5 \mathrm{sec}$ for 10 words.

The beginning of each trial was signaled by the printed word

Table 1

Examples of Test Sentences and Interpolated Words

Test Sentence:

Bananas and sour cream were served as a midnight snack.

10-Word Sentence:

When the cabin is empty, squirrels make nests in it.

Five 2-Word Sentences:

We won. Never delay. Carry on. We drifted. Faucets drip.

Random String:

Never In Shower Was Attack And Castle Him Mainly Rather
"READY." A test sentence was displayed, followed by 10 interpolated words. Subjects were instructed to read aloud all words that appeared in the window and to recall as accurately as possible the first (test) sentence when the recall signal appeared. Recall was signaled by a row of stars, at which point the drum was stopped. Subjects had as long as they needed for recall. It was emphasized that recall of the interpolated material would never be required, and that subjects should guess in recalling the test sentence, if necessary. Three practice trials were given, one with each type of interpolated material. Recall was tape recorded, and scoring was done from verbatim transcriptions. A semantic scoring criterion was used that allowed credit for changes of verb tense and number, substitution of synonyms, and reordering of words that did not alter the meaning. A verbatim criterion was also employed.

\section{Recall of Interpolated Material}

Subjects. The subjects were 14 additional introductory psychology students.

Materials and Design. Materials and design were the same as previously described.

Procedure. The experimental method was exactly the same as previously described, with the following exception. Subjects were instructed to recall the interpolated material as well as the test sentences. It was emphasized that they should always recall the test sentence before attempting to recall the interpolated material. Random words and two-word sentences could be recalled in any order.

Scoring of the interpolated material was based on the number of words recalled so that comparisons could be made across the three types of material. Word order was ignored in the random and 2-word sentence conditions; a paraphrase criterion was used for the 10-word sentences. Credit was given for the number of originally presented words conveyed by the recalled version.

\section{RESULTS}

Table 2 shows the proportion of test sentences in which the complete meaning was recalled for each of the interpolated task conditions. The left half shows performance when the interpolated material was only read but not recalled; the right half shows performance that was accompanied by recall of the interpolated material. A 2 by 3 analysis of variance for repeated measures was performed on each set separately.

No effects of structure or format were obtained when subjects read but did not recall the interpolated material. However, when recall of the interpolated material was required, both structure and format significantly affected recall of the test sentence $[F(2,65)=$ $4.97, \mathrm{p}<.01$, for structure; $\mathrm{F}(1,65)=5.71, \mathrm{p}<.05$, for format]. Newman-Keuls post hoc tests indicated that random words produced more interference than either 10-word sentences or 2-word sentences. Recall was superior in the simultaneous format condition, but it is clear from Table 2 that this main effect resulted primarily from the format effect when the interpolated material was a 10 -word sentence. This observation is confirmed by a significant Structure by Format interaction $[F(2,65)=3.87, p<.05]$. Post hoc comparisons indicated that the single 10 -word sentences produced more interference when presented 2 words at a time (successive format) than when all 10 words were presented at once (simultaneous format). In addition, the 
Table 2

Proportion of Test Sentences Recalled in Each Interpolated Task Condition

\begin{tabular}{cccccc}
\hline & \multicolumn{2}{c}{ No Recall* } & & \multicolumn{2}{c}{ Recall* } \\
\cline { 3 - 5 } \cline { 5 - 6 } Structure & $\begin{array}{c}\text { Simul- } \\
\text { taneous }\end{array}$ & $\begin{array}{c}\text { Succes- } \\
\text { sive }\end{array}$ & $\begin{array}{c}\text { Simul- } \\
\text { taneous }\end{array}$ & $\begin{array}{c}\text { Succes- } \\
\text { sive }\end{array}$ \\
\hline One 10-Word Sentence & .72 & .65 & .76 & .52 \\
Five 2-Word Sentences & .72 & .67 & .63 & .65 \\
10 Random Words & .63 & .64 & .54 & .49 \\
\hline
\end{tabular}

*Of interpolated material

Table 3

Mean Recall of Interpolated Words in Each Structure and Presentation Format

\begin{tabular}{ccc}
\hline & \multicolumn{2}{c}{ Format } \\
\cline { 2 - 3 } Structure & Simultaneous & Successive \\
\hline One 10-Word Sentence & 7.39 & 5.51 \\
Five 2-Word Sentences & 3.26 & 2.73 \\
10 Random Words & 1.51 & 2.02 \\
\hline
\end{tabular}

random strings (presented either simultaneously or successively) produced more interference than the single 10-word sentences presented in the simultaneous format. The five 2-word sentences were intermediate and did not differ significantly from any other condition.

When scored according to the verbatim criterion, the number of sentences perfectly recalled was $18 \%$; no significant effects of structure or format were obtained. The comparable figure for the procedure not requiring recall of the interpolated material was $23 \%$; again, no significant effects were found.

A comparison of the two procedures indicated that overall recall of the test sentences was not significantly reduced by the requirement to recall the interpolated words as well ( $68 \%$ vs. $60 \%)$. However, this finding is not particularly surprising if it resulted from a canceling out of the increased recall requirement by a reduction in the number of interpolated units (i.e., by chunking that was induced by the very recall requirement).

Recall of the interpolated materials was analyzed to measure chunking directly. The mean number of words recalled in each condition is presented in Table 3.

A 2 by 3 analysis of variance indicated main effects of structure $[F(2,26)=90.51, p<.01]$ and format $[F(1,13)=9.35, p<.01]$, and a Structure by Format interaction $[F(2,26)=9.99, p<.01]$. Newman-Keuls post hoc tests confirmed that the three structures differed significantly from each other. Regardless of presentation format, more interpolated words were recalled when they constituted a single 10 -word sentence than five 2 word sentences, which in turn were recalled better than when the words were unrelated (random string). Recall was higher when all interpolated words were presented simultaneously rather than successively. This effect was due primarily to the format effect when a single sentence constituted the interpolated material.

\section{DISCUSSION}

Results from the present studies support the conclusion that semantic processing is subject to disruption by a subsequent task and that it is sensitive to the information processing demands of that task. When subjects were instructed to recall interpolated material in addition to test sentences, the number of major syntactic units in the interpolated material determined the extent of disruption of test sentence processing. Two important points must be noted.

First, there were no effects on retention of verbatim information, suggesting that displacement from short-term storage occurs at the word level, replicating Jarvella's (1971) finding. That is, reading 10 additional words displaced verbatim information from short-term or working memory, regardless of the syntactic structure of those 10 words and regardless of the task requirement in effect.

The second point is that the syntactic structure of the interpolated material made a difference only when subjects were required to recall it along with the test sentence. This result is consistent with Aaronson and Shapiro's (1976) conclusion that subjects do not unitize on a syntactic basis unless recall is demanded. In the present case, we cannot conclude that subjects did not analyze syntactic structure when recall was not required, but, rather, that the structure was irrelevant to that task. Syntactic structure provides the basis for organizing rehearsal units in a recall task. Ten random words could not be further reduced into fewer units and produced the greatest amount of interference in the present study. Ten-word sentences, on the other hand, could be reduced to perhaps two units, resulting in little interference.

Analysis of recall of the interpolated material supports the unitization hypothesis. Inspection of the data in the simultaneous format condition shows that the number of words recalled from the 10-word sentences was over twice the number of words recalled from 2 -word sentences ( 7.39 vs. 3.26 ); likewise, the number recalled from 2-word sentences was over twice the number of random words recalled (3.26 vs. 1.51$)$. Thus, it appears that a constant number of units is retained, but the size of the units (or "chunks"; Miller, 1956) varies with the structure (one, two, or four words, approximately).

The robustness of syntactic unitization is evident in recall of the 10-word sentences presented in the successive format. Even though they produced as much interference as the random strings, they were recalled significantly better, suggesting subsequent integration into larger units. This effect plus the absence of a structure effect when recall was not required support Aaronson and Shapiro's (1976) contention that subjects adopt different coding strategies to meet task demands (see also Orasanu, 1975).

The present results indicate that memory for sentence meaning is not as impervious to disruption as implied by earlier studies. At least two interpretations of this finding can be offered. Either semantic analysis continues much longer than was previously thought and competes with a subsequent task for processing capacity, or the completed semantic representation is more fragile than was previously thought and subject to loss by a subsequent task. Further studies are needed to tease apart these alternatives.

Our findings raise a further question: To what extent was semantic disruption a function of the recall task demand? Those studies that have shown superior memory for sentence meaning have usually used recognition procedures (e.g., Orasanu, 1975). The present recall requirement may have induced "deeper" processing of test sentences (cf. Craik \& Lockhart, 1972), processing that might take longer to complete, thus setting the stage for disruption by the subsequent task. The effect of varying the task demands in the present study illustrates their importance in determining the nature of processing. 


\section{REFERENCES}

Aaronson, D., \& Shapiro, H. Performance theories for sentence coding: Some qualitative observations. Journal of Experimental Psychology: Human Perception and Performance, 1976, 2, 42-55.

Bransford, J. D., \& Johnson, M. C. Consideration of some problems of comprehension. In W. G. Chase (Ed.), Visual information processing. New York: Academic Press, 1973.

BRENER, L. R. An experimental investigation of memory span. Journal of Experimental Psychology, 1940, 26, 467-482.

Craik, F. I. M., \& LockharT, R. S. Levels of processing: A framework for memory research. Journal of Verbal Learning and Verbal Behavior, 1972, 11, 671-684.

Glanzer, M., Gianutsos, R., \& Dubin, S. The removal of items from short-term storage. Journal of Verbal Learning and Verbal Behavior, 1969, 8, 435-447.

JARVELlA, R. J. Syntactic processing of connected speech. Journal of Verbal Learning and Verbal Behavior, 1971, 10, 409-416.
Jarvella, R. J., Snodgrass, J. G., \& Adler, A. P. Memory for ongoing spoken discussion. In A. M. Lesgold, J. W. Pellegrino, S. D. Fokkema, \& R. Glaser (Eds.), Cognitive psychology and instruction. New York: Plenum, 1978.

KATZ, S. Role of instructions in abstraction of linguistic ideas. Journal of Experimental Psychology, 1973, 98, 79-84.

KINTSCH, W. The representation of meaning in memory. Hillsdale: N.J: Erlbaum, 1974.

Kintsch, W., \& BAtes, E. Recognition memory for statements from a classroom lecture. Journal of Experimental Psychology: Human Learning and Memory, 1977, 3, 150-159.

Mille r, G. A. The magic number seven, plus or minus two. Psychological Review, 1956, 63, 81-97.

Orasanu, J. The effect of encoding strategies on recognition memory for sentences. Unpublished doctoral dissertation, Adelphi University, 1975.

Sachs, J. S. Memory in reading and listening to disclosure. Memory \& Cognition, 1974, 2, 95-100.

(Received for publication August 15, 1979.) 